\title{
Interactions between the Lateral Habenula and the Hippocampus: Implication for Spatial Memory Processes
}

\author{
Romain Goutagny*,1,2, Michael Loureiro',2, Jesse Jackson ${ }^{3}, J^{1,2}{ }^{1,2 h}$ Chaumont $^{2,4}$, Sylvain Williams ${ }^{3}$, \\ Philippe Isope ${ }^{2,4}$, Christian Kelche ${ }^{1,2}$, Jean-Christophe Cassel ${ }^{1,2}$ and Lucas Lecourtier*, 1,2 \\ 'Laboratoire de Neurosciences Cognitives et Adaptatives, UMR7364, Centre National de la Recherche Scientifique (CNRS), Strasbourg, \\ France; ${ }^{2}$ Faculté de Psychologie, Université de Strasbourg, Strasbourg, France; ${ }^{3}$ Douglas Mental Health University Institute, McGill University, \\ Montréal, QC, Canada; ${ }^{4}$ Institut des Neurosciences Cellulaires et Intégratives, UPR3212, Centre National de la Recherche Scientifique \\ (CNRS), Strasbourg, France
}

\begin{abstract}
The lateral habenula $(\mathrm{LHb})$ is an epithalamic structure connected with both the basal ganglia and the limbic system and that exerts a major influence on midbrain monoaminergic nuclei. The current view is that $\mathrm{LHb}$ receives and processes cortical information in order to select proper strategies in a variety of behavior. Recent evidence indicates that LHb might also be implicated in hippocampus-dependent memory processes. However, if and how LHb functionally interacts with the dorsal hippocampus (dHPC) is still unknown. We therefore performed simultaneous recordings within $\mathrm{LHb}$ and $\mathrm{dHPC}$ in both anesthetized and freely moving rats. We first showed that a subset of LHb cells were phase-locked to hippocampal theta oscillations. Furthermore, LHb generated spontaneous theta oscillatory activity, which was highly coherent with hippocampal theta oscillations. Using reversible LHb inactivation, we found that LHb might regulate dHPC theta oscillations. In addition, we showed that LHb silencing altered performance in a hippocampus-dependent spatial recognition task. Finally, increased coherence between $\mathrm{LHb}$ and $\mathrm{dHPC}$ was positively correlated to the memory performance in this test. Collectively, these results suggest that $\mathrm{LHb}$ functionally interacts with the hippocampus and is involved in hippocampus-dependent spatial information processing. Neuropsychopharmacology (2013) 38, 24I8-2426; doi:10.1038/npp.2013.142; published online 26 June 2013
\end{abstract}

Keywords: theta oscillations; gamma oscillations; coherence; spatial memory

\section{INTRODUCTION}

The lateral habenula (LHb) is implicated in cortically driven functions such as the evaluation of motivational and reward values as well as the anticipation of motivational events (Bromberg-Martin et al, 2010; Matsumoto and Hikosaka, 2007, 2009), the selection of fear responses (Agetsuma et al, 2010; Okamoto et al, 2012), the integration of negative feedback information (Ullsperger and von Cramon, 2003), and attention (Lecourtier and Kelly, 2005). In addition, dysfunction of the habenula has been evidenced in psychiatric diseases, such as schizophrenia and depression (Hikosaka, 2010; Sartorius and Henn, 2007; Shepard et al, 2006). The current view is that LHb integrates information stemming from the basal ganglia and the limbic system to participate in the selection of appropriate behavioral strategies (Geisler and Trimble, 2008; Hikosaka, 2010).

More recently, a role in learning and memory has also been emphasized (Bromberg-Martin et al, 2010; Lecourtier

*Correspondence: Dr R Goutagny or Dr L Lecourtier, Laboratoire de Neurosciences Cognitives et Adaptatives, CNRS UMR7364, Université de Strasbourg, 12, rue Goethe, F-67000 Strasbourg, France,

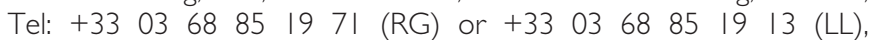
E-mail: goutagny@unistra.fr (RG) or l.lecourtier@unistra.fr (LL)

Received 20 February 2013; revised 3 May 2013; accepted 27 May 2013; accepted article preview online 5 June 2013 et al, 2004; Shumake et al, 2010). More precisely, lesion of the habenular complex induced spatial reference memory deficits in the water maze, during both acquisition and retention of the task (Lecourtier et al, 2004). These effects of habenular complex lesion mimic in multiple ways the results obtained after dorsal hippocampal (dHPC) lesion, indicating that LHb might functionally interact with hippocampal networks during spatial memory processes.

Information processing and storage by brain networks requires a high level of coordination between the multiple neuronal groups. One likely mechanism is through rhythmic activity of neuronal populations, which give rise to oscillations in local field potentials (Womelsdorf et al, 2007). Among these brain rhythms, the hippocampal theta oscillations have been described as a critical player in memory consolidation (Buzsaki, 2002). Disruption of theta rhythm results in spatial memory deficits (Winson, 1978) and restoration of theta-like rhythmicity restores learning capacities in rats (McNaughton et al, 2006). It is now well demonstrated that communication between brain areas involves phase synchronization (ie, coherence) of oscillations (Fries, 2005). Indeed, increases in theta coherence between the hippocampus and extra-hippocampal areas have been linked to memory performance in various tasks (Benchenane et al, 2010; Popa et al, 2010; Seidenbecher et al, 2003). 
Although evidence of interactions between LHb and dHPC is still missing, as there are no direct connections between the two structures, it is suggested by previous functional and electrophysiological studies. Using mapping of regional changes in brain metabolic activity, a functional correlation was shown to occur between LHb and dHPC during fear conditioning (Gonzalez-Pardo et al, 2012). Furthermore, electrical or iontophoretic activation of $\mathrm{LHb}$ modulates hippocampal pyramidal cell activity, mainly through a serotonergic relay residing in the median raphe nucleus (Zagami et al, 1995). Therefore, as functional coupling may occur between pairs of indirectly connected regions (Adachi et al, 2012; Bressler and Kelso, 2001; Leopold and Maier, 2012), LHb might be implicated in spatial memory processes through functional interactions with dHPC networks.

In the present study, using different experimental approaches ranging from in vivo electrophysiology to behavioral assessment of spatial memory processes, we show, for the first time, that LHb is functionally coupled with the dHPC and that this newly described corticosubcortical network might have a key role in spatial memory processes.

\section{MATERIALS AND METHODS}

\section{Animals and Surgery}

A total of 38 male Long-Evans rats $(250-350 \mathrm{~g})$ were used in compliance with the regulations specified by the European Committee Council Directive (86/609/EEC; authorization $\mathrm{n}^{\circ} 67-215$ for J-CC). They were housed individually in a quiet room, under a 12-h light/dark cycle (light on at 0700 hours) with ad libitum access to food and water.

For single-cell recordings, seven rats were anesthetized with urethane $(1.4 \mathrm{~g} / \mathrm{kg}$, i.p.) and placed in a stereotaxic frame. One extradural electrode located above the dHPC was used to record theta oscillations. The other, placed on the occipital crest, was used as a reference.

For freely moving recordings, 15 rats were anesthetized with sodium pentobarbital $(50 \mathrm{mg} / \mathrm{kg}$, i.p) and secured in a stereotaxic frame (Kopf, USA). Two tungsten microwire electrodes $\left(50 \mu \mathrm{m}\right.$; impedance $\sim 1 \mathrm{M}^{\circ}$ at $\left.1 \mathrm{KHz}\right)$ were positioned in LHb (anteroposterior (AP), - 3.5; mediolateral (ML), \pm 0.7 ; dorsoventral (DV), -4 from dura). Among those 15 rats, 10 were implanted with an additional electrode in the proximal stratum radiatum of region CA1 of the dHPC (AP, $-3.8 ; \mathrm{ML}, \pm 2.7$; DV, -2.3 from dura). Among those 10 rats, 4 were used to assess LHb-dHPC coherence during sleep-waking cycle and exploration of a known environment (see below) (LHb cells were recorded in 3 of those 4 rats) and 6 were used to assess LHb-dHPC coherence during the spatial recognition test (see below). Two screws were inserted in posterior and anterior portions of the skull as ground and reference, respectively. All electrodes and wires were fixed to the skull using SuperBond (Sun Medical Co., Shiga, Japan). They were soldered to a six-pin circular connector (Plastic one, USA) that was secured to the skull using acrylic cement.

Inactivation of LHb during the spatial recognition test was done in 12 rats bilaterally implanted with guide cannulas $1 \mathrm{~mm}$ above LHb. Finally, four rats were implanted with tungsten electrodes in the dHPC as described above, in addition to guide cannulas $1 \mathrm{~mm}$ above LHb in order to explore the effects of LHb inactivation on dHPC oscillations. Rats were allowed a 10-day post-operative rest before the experiments.

\section{LHb Inactivation and Spatial Recognition Test}

The test is adapted from Galani et al (1998). It was performed in an open field (OF; $45 \times 45 \times 30 \mathrm{~cm}$ ), including a spatial cue (black and white striped paper sheet, $29 \times 25 \mathrm{~cm}^{2}$ ) on an inner side wall. A paper sheet comprising a mosaic of squares of equivalent sizes $(4 \times 4)$ was placed under the clear Plexiglas floor of the OF in order to assess locomotor activity by numbering the crossings of the different squares. The training phase comprised six 6 -min sessions interspaced by 3 -min home cage resting periods. Sessions 1-3 represented the habituation to the OF. Then, three objects were placed in the OF and remained at the same location during sessions 4-6 (habituation to the objects). For session 7 (spatial recognition), one object was placed in a previously empty location ('new location' object), a second object was substituted for the one which became 'new location' ('substituted' object), and the third object was left where it was before ('undisplaced' object) (see Figure 5a for a description of the $\mathrm{OF}$ and the protocol). $\mathrm{LHb}$ inactivation was performed after session 6 by an infusion of the GABA-A agonist muscimol (Musc), as previously described (Winter et al, 2011). We used Musc to selectively inhibit $\mathrm{LHb}$, as GABA-A receptors are found in $\mathrm{LHb}$, whereas the medial habenula does not present GABAA receptor signaling (Wang et al, 2006). Musc ( $n=6$ rats; $0.25 \mu \mathrm{g} / \mu \mathrm{l}, 0.35 \mu \mathrm{l}$ per side; Sigma) dissolved in artificial cerebrospinal fluid (aCSF; in $\mathrm{mM}$ : $\mathrm{NaCl}, 145 ; \mathrm{KCl}, 2.7$; $\mathrm{MgCl}_{2}$ 1.0; and $\mathrm{CaCl}_{2} ; 1.2$ ) was infused in $\mathrm{LHb}$ over $1 \mathrm{~min}$; the injection cannulas were left in place for an additional $30 \mathrm{~s}$ before being retracted. Control rats $(n=6)$ were administered the same volume of aCSF at the same infusion rate. Session 7 was started 12 min post infusion. To assess spatial memory, the amount of time sniffing each object was compared for the two groups during session 7. In addition, a recognition index was calculated as follows: (('substituted' total sniffing time + 'new location' total sniffing time)/ ('substituted' total sniffing time + 'new location' total sniffing time + 'undisplaced' total sniffing time)).

\section{Electrophysiology}

Single cell recordings. In the presence of spontaneously occurring hippocampal theta, single units were recorded in $\mathrm{LHb}$ using glass microelectrodes (10-20 M 2 ) filled with $0.5 \mathrm{~m}$ sodium acetate and $2.5 \%$ neurobiotin. The signal was amplified, filtered between 0.1 and $5 \mathrm{kHz}$ and digitized at $15 \mathrm{kHz}$.

Freely moving recordings. Experiments began 10-14 days after surgery. Recordings were first carried out for $24 \mathrm{~h}$ across the spontaneous sleep-wake cycle. On the following day, rats were placed in the same OF used for electrophysiological recordings for $30 \mathrm{~min}$ per day for 3 days. LFPs were recorded on the third day to collect data regarding the 'exploration of a known environment' condition. Animals 
were connected via a rotating commutator (Plastic one; USA) to individual animal bioamps (AD instruments, UK). LFPs were amplified $(1000 \times)$, filtered $(0.1-500 \mathrm{~Hz})$, and digitized at $1000 \mathrm{~Hz}$. For cell recordings, the signal was filtered between 0.3 and $10 \mathrm{KHz}$ and sampled at $20 \mathrm{KHz}$.

Recordings during the spatial recognition test. The same electrophysiological set up was used and recordings were performed on six rats during the spatial recognition test.

Data analysis. All the analyses were performed using custom-made scripts in Matlab (MathWorks, USA).

Sleep-wake cycle. Sleep stages were determined first using visual scoring and a hypnogram was created based upon the following characteristics: wake (W) was characterized by an activated low-amplitude field activity; and slow wave sleep (SWS) was clearly distinguished by highvoltage slow waves and spindles and a regular and pronounced theta rhythm signaled the onset of rapid eye movement sleep (REM or paradoxical sleep). To confirm this visual scoring, we used a recently described algorithm for behavioral state classification based on the analysis of LFPs (Supplementary Figure S1, (Gervasoni et al, 2004)).

Spectral analysis. Power spectra and spectral coherence were calculated using the Chronux signal processing toolbox (Bokil et al, 2010) with a time-frequency product of three and five tapers. Coherence values above the $95 \%$ confidence interval were considered significant. For timefrequency analysis, we used a 4-s window and moved across the data in 1-s increments. Power (integrated band power for theta; $6-9 \mathrm{~Hz}$ and gamma, $30-100 \mathrm{~Hz}$ ) and coherence (mean coherence over the specified frequency bands) were calculated in 4 -s bins over at least 4 periods of $2 \mathrm{~min}$ for each vigilance state.

The phase locking value (PLV) between LHb and dHPC was quantified using at least 2 min of continuous data and was computed as previously described (Gu et al, 2013). Briefly, we calculated the mean resultant length (CircStat, toolbox; (Berens, 2009) of the phase difference between the two LFPs. A value of 1 indicates perfect phase synchrony. To test the significance of the PLV, we generated a surrogate set of 200-400 PLVs by shuffling the data between $2 \mathrm{~s}$ and the length of the entire signal used for the original analysis. The original PLV was required to be greater than the mean +2 SD of this surrogate shuffled data set in order to be considered statistically significant.

During the spatial recognition test, power spectra and spectral coherence were assessed during each session.

Extracellular spiking analysis. Single units were sorted using the principal component analysis on Spike2 software (CED, UK) based on spike waveform. Phase locking to theta and gamma rhythm was then assessed using Matlab (Supplementary Materials and Methods).

Histology. Electrodes and guide cannulas placements were verified using cresyl violet staining on $40 \mu \mathrm{m}$-thick brain slices. Muscimol infusion sites were identified using microinjections of fluorescent muscimol (Invitrogene,
USA); $40 \mu \mathrm{m}$-thick slices were observed under a microscope at a wave length of $544 \mathrm{~nm}$.

\section{Statistical Analyses}

All data are expressed as mean \pm SEM. Statistical analyses used Graphpad (Prism). Paired $t$-tests were used to compare two means. For multiple-group analyses, two-way ANOVA and Bonferroni's post-hoc test were used. For analyzing spatial recognition data, we used a Group $\times$ Object ANOVA, followed by a Newman-Keuls post-hoc test. Activity measures during session 7 were compared using paired $t$-tests.

\section{RESULTS}

\section{Spontaneous Activity in LHb}

Recent data indicate that $\mathrm{LHb}$ might have a role in hippocampus-dependent memory processes (Lecourtier et al, 2004). However, evidence of functional interactions between the $\mathrm{LHb}$ and hippocampus is still missing. To determine whether $\mathrm{LHb}$ cell firing was modulated by ongoing hippocampal oscillatory activity, we performed single-cell extracellular recordings using glass microelectrodes in urethane-anesthetized animals. A sample of 15 neurons was recorded in LHb ( $n=7$ rats) during at least one complete brain state alternation (LIA and theta state; (Clement et al, 2008)). LHb neurons could be segregated into two sub-groups according to their firing rate during brain state alternations. Neurons from the first subgroup $(n=7)$ were characterized by a tonic and regular discharge pattern but did not show any modification of their firing rate between the different brain states $(11.3 \pm 5.9 \mathrm{~Hz}$ during LIA $v s 10.1 \pm 5.6 \mathrm{~Hz}$ during theta, $t_{6}=1.878 ; p>0.1$, paired $t$-test; Figure $1 \mathrm{a})$. The second group $(n=8)$ contained neurons exhibiting a significant tonic increase in their mean firing rate during theta states compared with LIA states $(6.3 \pm 1.8 \mathrm{~Hz}$ during LIA $v s 11.6 \pm 2.4 \mathrm{~Hz}$ during theta, $t_{8}=6.129 ; p<0.001$, paired $t$-test; Figure $\left.1 \mathrm{~b}\right)$. They were therefore classified as theta-on cells (Colom and Bland, 1987). These results indicate that LHb contains a subpopulation of neurons whose firing rate is closely related to hippocampal activity. However, under urethane anesthesia, the hippocampus only exhibits atropine-sensitive theta (or type 2 theta, (Kramis et al, 1975)). To further determine whether LHb cell firing was related to dHPC oscillatory activity in freely moving animals (with the presence of atropine-resistant hippocampal theta or type 1 theta), we were able to record cells within LHb during a complete sleep and waking cycle ( $n=7$ cells in 3 rats) together with local field potential recordings in the dHPC in non-anesthetized animals. As anticipated with the results from the urethaneanesthetized animals, LHb cells were more active during theta oscillations (both during active wake and REM) than during slow-wave sleep $(7.913 \pm 3.19 \mathrm{~Hz}$ during Wake, $6.552 \pm 3.18 \mathrm{~Hz}$ for SWS and $9.33 \pm 3.5 \mathrm{~Hz}$ for REM, $\mathrm{F}_{2,6}=4.884 ; p<0.05$, one-way ANOVA; Figure $2 \mathrm{~b}$ ). Among those cells, three exhibit a clear bimodal distribution in the inter-spike interval histogram (Figure 2d), with the peaks located at gamma $(25 \mathrm{~ms}, 40 \mathrm{~Hz})$ and theta $(115 \mathrm{~ms}, 8.5 \mathrm{~Hz})$ period. In addition, these cells were phase-locked to dHPC theta but not gamma oscillations (Figure 2e). Therefore, a 
subset of LHb cells maintains a close phase relationship with dHPC theta oscillations.

The presence of theta frequency firing suggests that $\mathrm{LHb}$ might spontaneously generate theta oscillations. We therefore performed LFP recordings within LHb ( $n=9$ rats) during natural sleep-waking cycle and exploration of a familiar environment. We found that LHb displayed marked theta oscillations during both exploration and rapid-eye movement sleep (REM; Figures $2 \mathrm{f}$ and g). The spectral power of LHb theta oscillations was not different between REM and exploration (18 $\pm 2.7 v s 15 \pm 2 \times 10^{-3} \mathrm{mV}^{2}$ for REM and exploration, respectively, $t_{8}=1.203 ; p>0.05$; Figure $2 \mathrm{~h}$ ). In accordance with LHb cells discharge pattern, we also detected the presence of prominent gamma rhythms in LHb (Figure 2g, inset). Gamma power was significantly higher during exploration than during REM $(4.52 \pm 0.6 v s$ $6.3 \pm 0.8 \times 10^{-3} \mathrm{mV}^{2}$ for REM and exploration, respectively, $t_{8}=3.051 ; p<0.05$, paired $t$-test; Figure $2 \mathrm{~h}$ ). Taken together, these data clearly indicate that LHb exhibits spontaneous oscillatory activity in the theta and gamma range that cannot be explained by volume conduction from the overlying hippocampus (Supplementary Figure S2). In addition, $\mathrm{LHb}$ activity maintains a close relationship with the dHPC.

\section{Interactions Between LHb and dHPC}

To further determine whether $\mathrm{LHb}$ and the dHPC were functionally related, we performed simultaneous LFP recordings in LHb and dHPC $(n=4)$ during the natural sleep-wake cycle and during the exploration of a familiar environment.

LHb and dHPC LFPs exhibited strong and significant coherence at theta rhythm during both REM and exploration (theta band coherence: $0.92 \pm 0.01$ during REM $v s$ $0.84 \pm 0.03$ during exploration; Figure $3 \mathrm{~b}$, left). To determine whether this theta coherence was related to dHPC theta power, we analyzed the phase locking value (PLV) between LHb and dHPC LFPs. A comparable PLV was also observed (theta PLV: $0.91 \pm 0.01$ during REM vs $0.78 \pm 0.06$ during exploration), suggesting that high spectral coherence was mediated by high phase-phase synchrony and not power alone (Figure $3 \mathrm{~b}$, right). As LHb LFPs exhibit gamma oscillations, we also computed the coherence at gamma rhythm between LHb and dHPC (Figure 3b). We found that dHPC and LHb maintained a significant albeit low gamma coherence during both behavioral states (gamma band coherence: $0.45 \pm 0.07$ during REM vs $0.49 \pm 0.1$ during exploration).

In addition, we calculated the phase lag between LHb and dHPC theta oscillations during both REM sleep and exploration of a known environment (Figures $3 c$ and $d$ ). In accordance with the coherence and PLV analysis, we showed that LHb theta oscillations maintain a highly stable phase relationship with dHPC theta activity.

\section{Effect of LHb Inactivation on dHPC Theta Oscillations}

As LHb and dHPC theta oscillations were highly phaselocked, we then assessed whether LHb might regulate dHPC oscillatory activity (Hangya et al, 2009). To this end, we recorded LFPs within dHPC following bilateral LHb inactivation in four rats. They consecutively received Musc and aCSF with a 24-h delay between each treatment. We computed dHPC theta and gamma power as well as theta peak frequency during active wake in the 10-20 min period following Musc $(0.25 \mu \mathrm{g} / \mu \mathrm{l}, 0.35 \mu \mathrm{l}$ per side) or aCSF infusion. We found that $\mathrm{LHb}$ inactivation led to a reduction in the predominant frequency of theta oscillations $(7.11 \pm 0.11 \mathrm{~Hz}$ following aCSF $v s 6.29 \pm 0.32 \mathrm{~Hz}$ following Musc, $t_{3}=3.756 ; p<0.05$, paired $t$-test; Figure $4 \mathrm{c}$ middle). The amplitude of theta oscillations was not changed following Musc injection compared with aCSF $\left(t_{3}=1.71\right.$; $p>0.1$, paired $t$-test) despite a tendency to decrease $\left(0.26 \pm 0.05 \mathrm{mV}^{2}\right.$ after aCSF vs $0.16 \pm 0.02 \mathrm{mV}^{2}$ after Musc; Figure 4c left). However, no effect was seen on hippocampal
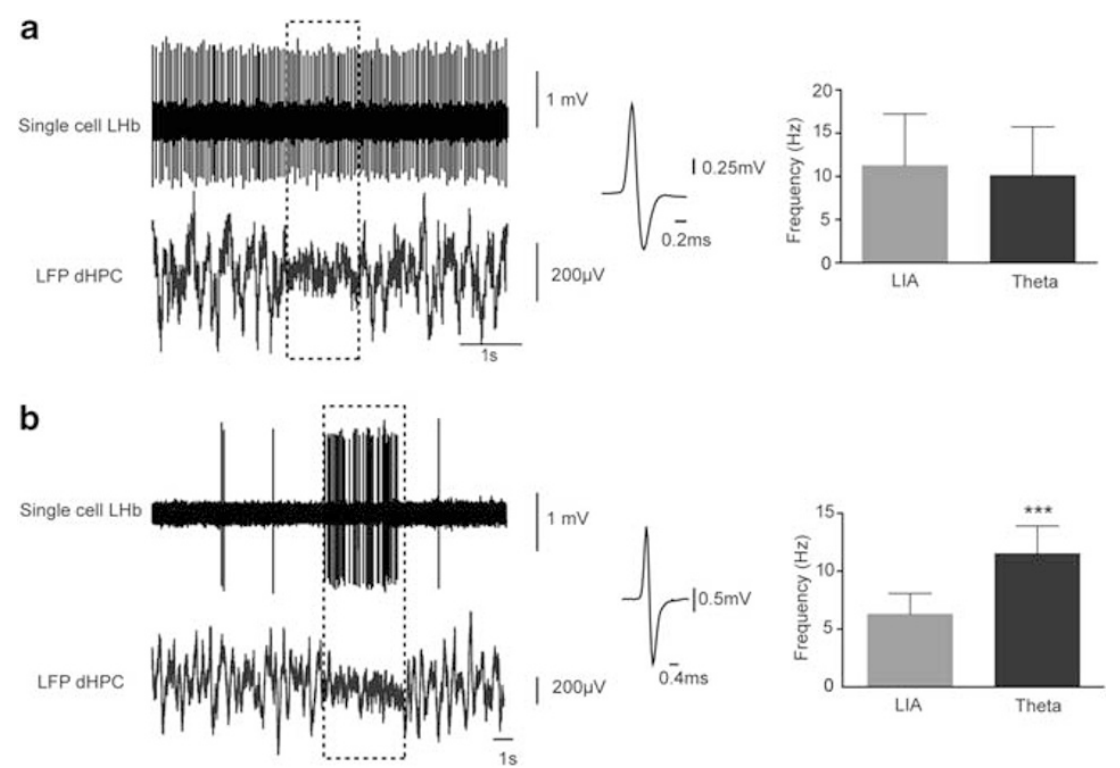

Figure I LHb neuronal firing in urethane anaesthetized animals. (a) Raw data (left) and mean firing rate (right) of unrelated LHb cells. These cell are active during both LIA and theta states (dashed rectangle). (b) Raw data (left) and mean firing rate (right) of theta-on cells in LHb. Note the selective increase in firing rate during theta state (dashed rectangle; $* * * * 0<0.00$ I). 
a

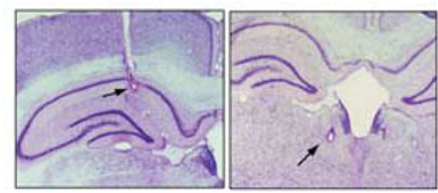

b

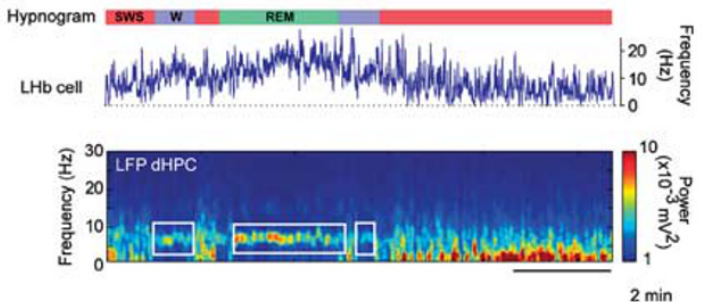

e

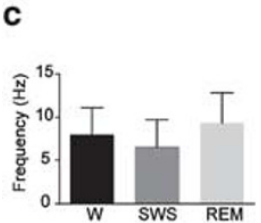

d

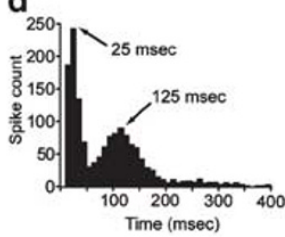

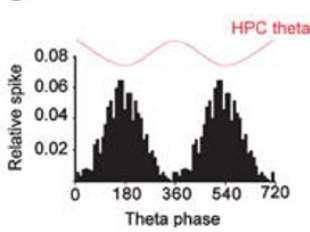

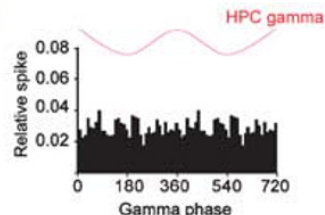

Gamma phase

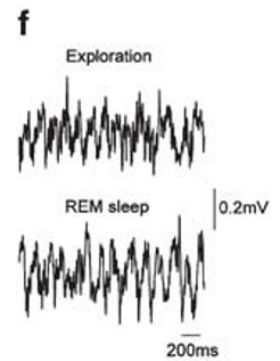

g
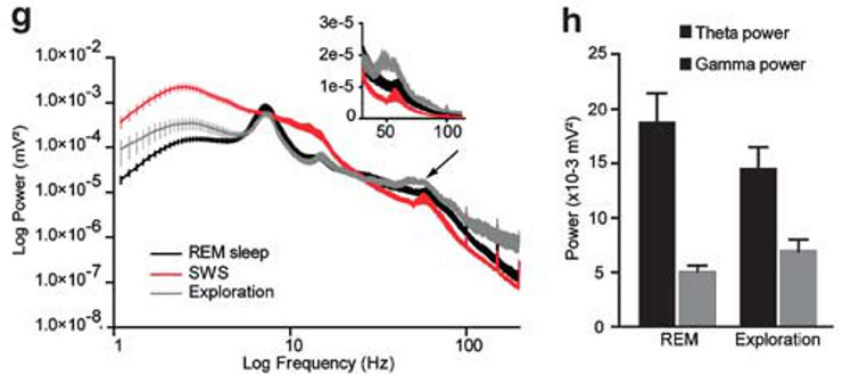

Figure 2 Discharge and oscillatory activity of LHb neurons across sleep-wake states. (a) Location of the recording electrode within LHb and dHPC (arrows). (b) Discharge of $\mathrm{LHb}$ neurons across sleep-wake states. Note the selective increase in frequency during $\mathrm{W}$ and REM (theta state, highlighted in the dHPC spectrogram). (c) Mean firing rate of LHb neurons across sleep-wake cycle. (d) Inter-spike interval histogram of one LHb neuron illustrating the bimodal distribution with the peaks located at gamma $(25 \mathrm{~ms}, 40 \mathrm{~Hz})$ and theta period $(\mathrm{II} 5 \mathrm{~ms}, 8.5 \mathrm{~Hz})$. (e) Cells in LHb exhibits phase relationship with dHPC theta oscillations but no gamma oscillations. ( $f$ ) Raw LFP trace recorded in LHb during REM and exploration. (g) LHb mean power spectrum ( $n=9$ rats) during REM, slow wave sleep and exploration of a familiar environment. Note the peak at theta frequency during REM and exploration, and the presence of sustained gamma activity (inset). (h) LHb mean theta and gamma power during REM and exploration of a familiar environment.

gamma oscillation (Figure 4c right). Collectively, these results suggest that $\mathrm{LHb}$ might specifically regulate hippocampal theta oscillations.

\section{Effect of LHb Inactivation on Recognition Memory}

We further assessed the potential role of LHb in hippocampus-dependent behavior by exploring the consequences of LHb inhibition during a spatial recognition test requiring intact hippocampal functioning (Figure 5a; Galani et al, 1998). Rats were treated with Musc ( $n=6$ rats) or aCSF ( $n=6$ rats) within LHb before session 7 . We found that LHb inhibition led to marked memory deficits as the ANOVA showed a significant Group $\times$ Object interaction $\left(\mathrm{F}_{2,20}=11.43 ; \quad p<0.001 ;\right.$ Figure $\left.5 \mathrm{~b}\right)$. Post-hoc analyses showed that during session 7 aCSF-treated rats spent significantly more time sniffing the 'new location' object $(p<0.01$ and $p<0.01$ as compared with the 'undisplaced' and 'substituted' objects, respectively), whereas Musctreated rats spent an equivalent sniffing time on the three objects (Figure 5b). Analysis of the recognition index showed a significant difference between the two groups $\left(t_{10}=5.00 ; p<0.001\right.$; Figure $\left.5 \mathrm{~d}\right)$; whereas aCSF-treated rats performed significantly above chance level (ie, 0.66; $p<0.05)$, Musc-treated rats did not. These results are unlikely to reflect a bias due to object characteristics or Musc-induced lack of motivation or exploratory behavior (see Supplementary Figure S3).
Finally, we aimed to determine whether LHb-dHPC coherence might predict memory performance. To this end, we recorded LFPs within LHb and dHPC during the spatial recognition test ( $n=6$ rats). As expected, all rats detected the spatial change of the objects and performed significantly above chance level (mean recognition index $\left.=0.7817 \pm 0.037 ; t_{5}=7.559, p<0.001\right)$. In addition, LHb-dHPC coherence during session 7 was linearly correlated with performance $\left(\mathrm{F}_{1,4}=10.63, p<0.05\right.$, Figure $\left.5 \mathrm{~d}\right)$. Furthermore, two distinct sub-groups could be identified based on memory performance. Interestingly, rats performing significantly better also exhibit significantly higher LHb-dHPC theta coherence during the test (Supplementary Figure S4). Altogether, our data indicate that LHb-dHPC coherence might have a key role in hippocampus-dependent spatial memory processing.

\section{DISCUSSION}

The major findings of the present study are that LHb cell firing is related to hippocampal theta oscillations and that LHb and dHPC might be functionally coupled through theta oscillatory activity. Furthermore, LHb influences dHPC theta oscillations, and LHb inactivation alters performance in a hippocampus-dependent spatial recognition task. Finally, LHb-dHPC coherence is directly related to memory performance. Together, these findings clearly indicate that 

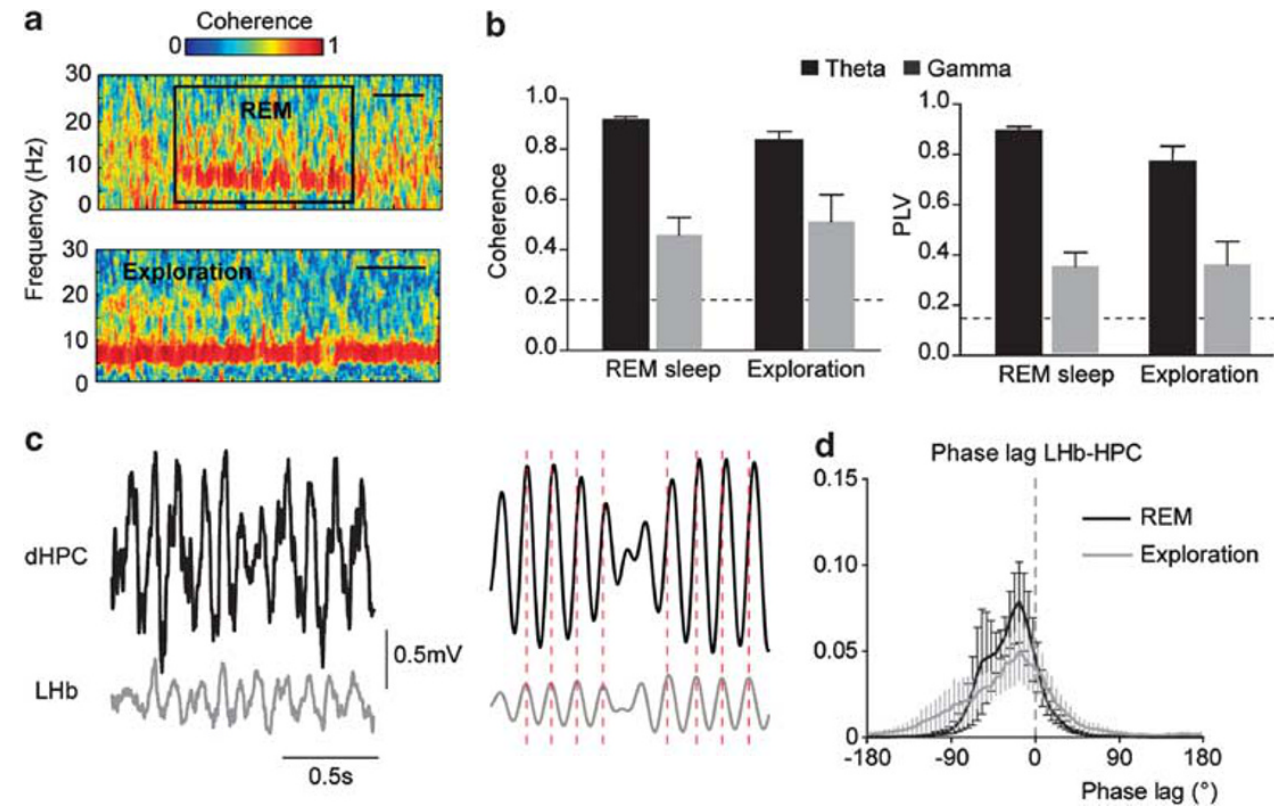

Figure 3 Interactions between LHb and dHPC. (a) Cohereogram illustrating theta coherence dynamics between LHb and dHPC during REM (upper panel) and exploration of a familiar environment. Bar = I min. (b) Mean theta and gamma coherence (left) or PLV (right) between LHb and dHPC. Note the similar pattern between the two analyses. (c) Raw (left) and theta filtered (right) traces simultaneously acquired in dHPC and LHb. (d) Mean phase-lag between $\mathrm{LHb}$ and $\mathrm{dHPC}$. Note that LHb theta oscillations present a clear phase-advanced relative to dHPC theta rhythm.
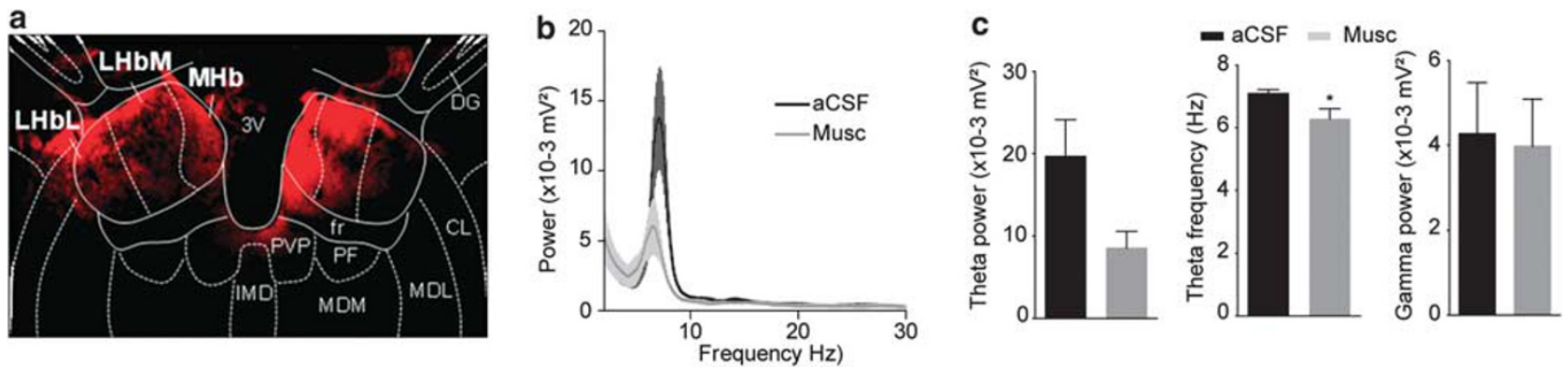

Figure 4 Reversible inactivation of LHb induces hippocampal theta alterations. (a) Representative photograph of the diffusion of fluorescent Muscimol within LHb. (b) Power spectrum analysis of dHPC oscillatory activity following aCSF or Musc infusion within LHb. (c) LHb inactivation through Musc infusion $(n=4$ rats) induces decrease in hippocampal theta power and frequency $(* 00.05)$ but had no effect on hippocampal gamma power.

LHb is part of a cortical/subcortical network involved in spatial information processing.

It is well known that spatial memory processes require intact hippocampal functioning (see (Frankland and Bontempi, 2005) for review). Therefore, the fact that habenular complex lesion induced marked spatial memory impairment (Lecourtier et al, 2004) indicates that LHb and dHPC might interact at some point during encoding and/or retrieval of a spatial memory. However, how these two structures are functionally interacting, despite the lack of direct physical connection, remains to be established. One suggested mechanism for such interaction is through neuronal synchronization by brain oscillations (Womelsdorf et al, 2007). By combining multiple experimental approaches, we showed that LHb and dHPC might form a functionally connected network structured through theta oscillatory activity. The observed strong theta coherence between LHb and dHPC suggests that these regions are functionally connected and are likely to exchange relevant information related to a particular behavioral context.

To our knowledge, it is the first time that oscillatory activity has been described within LHb in freely moving animals. One issue could reside in the fact that recorded LFPs can arise from volume conduction of distant signals (Kajikawa and Schroeder, 2011). However, the fact that cellular activity within LHb is phase-locked to hippocampal theta oscillations and that theta rhythm is still present in $\mathrm{LHb}$ after local referencing (Supplementary Figure S2) suggests that LHb activity is theta modulated. LHb theta oscillations are likely generated through the influence of an external pacemaker.

Given the important projection from the medial septum/ diagonal band of Broca complex (MS/DBB; (Tomimoto et al, 1987), it is likely that LHb activity is, in part, modulated by septal afferences, which also represent a strong dHPC theta modulator (Bland et al, 1996; Goutagny et al, 2008). However, it is unlikely that LHb only represents 
a OF Habituation (session 1-3)

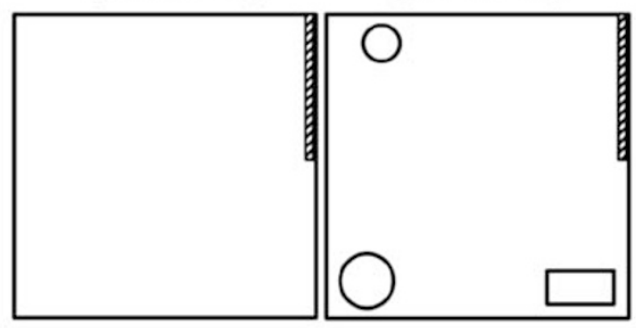

b

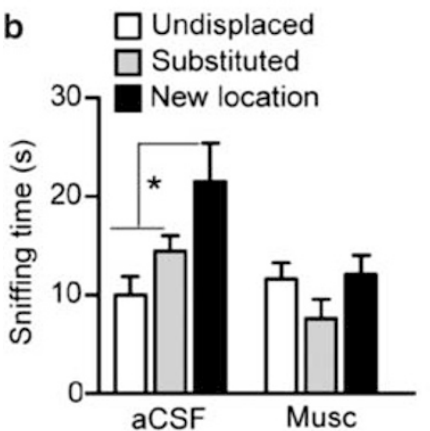

Objects Habituation (session 4-6)

C

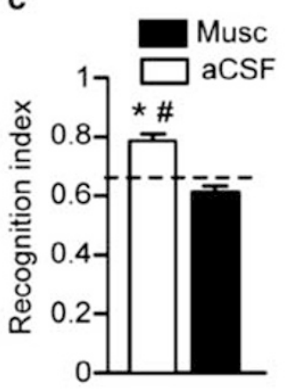

Spatial recognition (session 7)

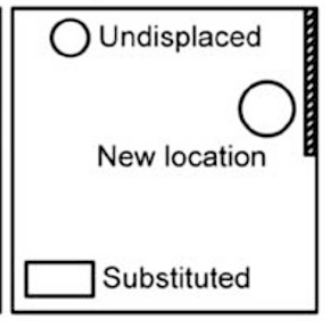

d

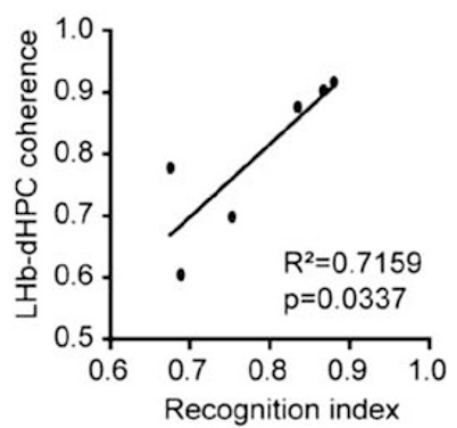

Figure 5 Reversible inactivation of LHb induces spatial recognition deficits. (a) Representation of the spatial recognition test used and adapted from Galani et al (1998). (b) During session 7, whereas control rats spent significantly more time sniffing the 'new location' object (* $p<0.0$ I vs 'substituted' and 'undisplaced'), Musc-treated rats spent an equivalent amount of time sniffing the three objects. (c) The recognition index showed that Musc-treated rats performed significantly worse than control rats during session 7 ( $p<0.0$ I vs Musc-treated rats; \#p<0.05 vs chance level, ie, 0.66). (d) In addition, when LHb and $\mathrm{AHPC}$ field potentials are simultaneously recorded during the test, their coherence during session 7 is linearly correlated with performance.

a relay station of information arising from the MS/DBB complex and transmitted to the dHPC. One hypothesis is that $\mathrm{LHb}$ is processing different information stemming from not only the septal area, but also the basal ganglia, as already shown (Hong and Hikosaka, 2008), or from prefrontal cortical areas (see Geisler and Trimble, 2008).

In addition, the fact that selective LHb inactivation reduced the frequency of $\mathrm{AHPC}$ theta oscillation is particularly important, as, to date, only the supramammillary (SuM) nucleus was known to modulate hippocampal theta frequency (Pan and McNaughton, 2004). Anatomically, although there are no direct connections between LHb and $\mathrm{dHPC}$, LHb projects to SuM. Therefore, it is possible that $\mathrm{LHb}$ influences SuM in order to modulate dHPC theta frequency. Such tuning might also occur through a modulation of dopamine release. Indeed, a hyperdopaminergic state, which has been shown to occur following $\mathrm{LHb}$ inactivation (Lecourtier et al, 2008), also induces a reduction in dHPC theta frequency (Dzirasa et al, 2009).

Furthermore, in our study, LHb inactivation led to marked deficits in a hippocampus-dependent spatial recognition task (Galani et al, 1998). This object-based task requires animals to focus their attention in order to collect information about the configuration of their environment. This information is then memorized and subsequently used to detect spatial changes during a test session taking place shortly after the last habituation session. We found that, during the test session, the level of coherence between LHb and dHPC at theta frequencies was positively correlated with the level of performance of the rats. This suggests that
LHb belongs to the network implicated in the processing of contextual information. However, the exact mechanisms responsible for the memory deficits observed after LHb inactivation still need to be clarified. One can hypothesize that the musc-induced memory deficits might arise from alterations, within dHPC, of key monoaminergic transmissions modulated by LHb, including dopamine which has a key role in dHPC-dependent short-term memory (Lisman et al, 2011). Also, LHb inactivation is likely to result in altered monoaminergic transmission within the prefrontal cortex (PFC) and consequently disturb PFC-dependent processes such as short-term memory and attention (Kesner and Churchwell, 2011; Robbins and Arnsten, 2009). As said above, and postulated by others (Geisler and Trimble, 2008), LHb possibly receives information from the basal ganglia, the septal area and PFC regions in order to select the proper strategy. Those information might be related not only to spatial configuration but also to goaldirected motor planning, attention, reaction to stress, and behavioral flexibility (see also Hikosaka, 2010).

In conclusion, this study shows that LHb belongs to the cortical-subcortical network implicated in the regulation of dHPC theta rhythm and spatial memory processes. Given the increasing evidence that LHb is involved in cortically driven behaviors, this study strongly suggests that LHb is in an ideal position not only to integrate and transmit cortical information to monoaminergic areas of the midbrain but also to generate a feedback to cortical structures in order to provide fine tuning of neural synchrony. Finally, our data suggest that $\mathrm{LHb}$ dysfunction in pathologies such as depression (Sartorius and Henn, 2007) participates in the 
genesis of deficits of cortically mediated functions through the alterations of cortical networks.

\section{FUNDING AND DISCLOSURE}

This work was supported by the University of Strasbourg and the Centre National de la Recherche Scientifique (CNRS). LL is supported by an ERA-NET NEURON grant (SuppHab). RG is supported by grants from the Fondation Fyssen, the European Research Executive Agency and the NARSAD. PI is supported by grant from the Agence Nationale de la Recherche (ANR-2010-JCJC-1403-1 MicroCer). The authors declare no conflict of interest.

\section{ACKNOWLEDGEMENTS}

We are grateful to Dr Okihide Hikosaka for his comments and suggestions. We are thankful to O. Bildstein, D. Egesi, and G. Edomwonyi for their assistance in animal care and to Dr Kwamivi Dzahini for providing the aCSF.

\section{REFERENCES}

Adachi Y, Osada T, Sporns O, Watanabe T, Matsui T, Miyamoto K et al (2012). Functional connectivity between anatomically unconnected areas is shaped by collective network-level effects in the macaque cortex. Cereb Cortex 22: 1586-1592.

Agetsuma M, Aizawa H, Aoki T, Nakayama R, Takahoko M, Goto $\mathrm{M}$ et al (2010). The habenula is crucial for experiencedependent modification of fear responses in zebrafish. Nat Neurosci 13: 1354-1356.

Benchenane K, Peyrache A, Khamassi M, Tierney PL, Gioanni Y, Battaglia FP et al (2010). Coherent theta oscillations and reorganization of spike timing in the hippocampal- prefrontal network upon learning. Neuron 66: 921-936.

Berens P (2009). CircStat: a MATLAB toolbox for circular statistics. J Stat Software 31: 1-21.

Bland BH, Trepel C, Oddie SD, Kirk IJ (1996). Intraseptal microinfusion of muscimol: effects on hippocampal formation theta field activity and phasic theta-ON cell discharges. Exp Neurol 138: 286-297.

Bokil H, Andrews P, Kulkarni JE, Mehta S, Mitra PP (2010). Chronux: a platform for analyzing neural signals. $J$ Neurosci Methods 192: 146-151.

Bressler SL, Kelso JA (2001). Cortical coordination dynamics and cognition. Trends Cogn Sci 5: 26-36.

Bromberg-Martin ES, Matsumoto M, Nakahara H, Hikosaka O (2010). Multiple timescales of memory in lateral habenula and dopamine neurons. Neuron 67: 499-510.

Buzsaki G (2002). Theta oscillations in the hippocampus. Neuron 33: 325-340.

Clement EA, Richard A, Thwaites M, Ailon J, Peters S, Dickson CT (2008). Cyclic and sleep-like spontaneous alternations of brain state under urethane anaesthesia. PLoS One 3: e2004.

Colom LV, Bland BH (1987). State-dependent spike train dynamics of hippocampal formation neurons: evidence for theta-on and theta-off cells. Brain Res 422: 277-286.

Dzirasa K, Santos LM, Ribeiro S, Stapleton J, Gainetdinov RR, Caron MG et al (2009). Persistent hyperdopaminergia decreases the peak frequency of hippocampal theta oscillations during quiet waking and REM sleep. PLoS One 4: e5238.

Frankland PW, Bontempi B (2005). The organization of recent and remote memories. Nat Rev 6: 119-130.
Fries P (2005). A mechanism for cognitive dynamics: neuronal communication through neuronal coherence. Trends Cogn Sci 9: $474-480$.

Galani R, Weiss I, Cassel JC, Kelche C (1998). Spatial memory, habituation, and reactions to spatial and nonspatial changes in rats with selective lesions of the hippocampus, the entorhinal cortex or the subiculum. Behav Brain Res 96: 1-12.

Geisler S, Trimble M (2008). The lateral habenula: no longer neglected. CNS Spectr 13: 484-489.

Gervasoni D, Lin SC, Ribeiro S, Soares ES, Pantoja J, Nicolelis MA (2004). Global forebrain dynamics predict rat behavioral states and their transitions. J Neurosci 24: 11137-11147.

Gonzalez-Pardo H, Conejo NM, Lana G, Arias JL (2012). Different brain networks underlying the acquisition and expression of contextual fear conditioning: a metabolic mapping study. Neuroscience 202: 234-242.

Goutagny R, Manseau F, Jackson J, Danik M, Williams S (2008). In vitro activation of the medial septum-diagonal band complex generates atropine-sensitive and atropine-resistant hippocampal theta rhythm: an investigation using a complete septohippocampal preparation. Hippocampus 18: 531-535.

Gu N, Jackson J, Goutagny R, Lowe GC, Manseau F, Williams S (2013). NMDA dependent phase synchronization between septal and temporal CA3 hippocampal networks. J Neurosci 33: 8276-8287.

Hangya B, Borhegyi Z, Szilagyi N, Freund TF, Varga V (2009). GABAergic neurons of the medial septum lead the hippocampal network during theta activity. J Neurosci 29: 8094-8102.

Hikosaka O (2010). The habenula: from stress evasion to valuebased decision-making. Nat Rev 11: 503-513.

Hong S, Hikosaka O (2008). The globus pallidus sends rewardrelated signals to the lateral habenula. Neuron 60: 720-729.

Kajikawa Y, Schroeder CE (2011). How local is the local field potential? Neuron 72: 847-858.

Kesner RP, Churchwell JC (2011). An analysis of rat prefrontal cortex in mediating executive function. Neurobiol Learn Mem 96: $417-431$.

Kramis R, Vanderwolf CH, Bland BH (1975). Two types of hippocampal rhythmical slow activity in both the rabbit and the rat: relations to behavior and effects of atropine, diethyl ether, urethane, and pentobarbital. Exp Neurol 49(1 Pt 1): $58-85$.

Lecourtier L, Defrancesco A, Moghaddam B (2008). Differential tonic influence of lateral habenula on prefrontal cortex and nucleus accumbens dopamine release. Eur J Neurosci 27: $1755-1762$.

Lecourtier L, Kelly PH (2005). Bilateral lesions of the habenula induce attentional disturbances in rats. Neuropsychopharmaco$\log y$ 30: 484-496.

Lecourtier L, Neijt HC, Kelly PH (2004). Habenula lesions cause impaired cognitive performance in rats: implications for schizophrenia. Eur J Neurosci 19: 2551-2560.

Leopold DA, Maier A (2012). Ongoing physiological processes in the cerebral cortex. Neuroimage 62: 2190-2200.

Lisman J, Grace AA, Duzel E (2011). A neoHebbian framework for episodic memory; role of dopamine-dependent late LTP. Trends Neurosci 34: 536-547.

Matsumoto M, Hikosaka O (2007). Lateral habenula as a source of negative reward signals in dopamine neurons. Nature 447: 1111-1115.

Matsumoto M, Hikosaka O (2009). Representation of negative motivational value in the primate lateral habenula. Nat Neurosci 12: $77-84$.

McNaughton N, Ruan M, Woodnorth MA (2006). Restoring thetalike rhythmicity in rats restores initial learning in the Morris water maze. Hippocampus 16: 1102-1110.

Okamoto H, Agetsuma M, Aizawa H (2012). Genetic dissection of the zebrafish habenula, a possible switching board for selection 


\section{LHb and hippocampal networks interactions}

R Goutagny et al

of behavioral strategy to cope with fear and anxiety. Dev Neurobiol 72: 386-394.

Pan WX, McNaughton N (2004). The supramammillary area: its organization, functions and relationship to the hippocampus. Progr Neurobiol 74: 127-166.

Popa D, Duvarci S, Popescu AT, Lena C, Pare D (2010). Coherent amygdalocortical theta promotes fear memory consolidation during paradoxical sleep. Proc Natl Acad Sci USA 107: 6516-6519.

Robbins TW, Arnsten AF (2009). The neuropsychopharmacology of fronto-executive function: monoaminergic modulation. Annu Rev Neurosci 32: 267-287.

Sartorius A, Henn FA (2007). Deep brain stimulation of the lateral habenula in treatment resistant major depression. Med Hypotheses 69: 1305-1308.

Seidenbecher T, Laxmi TR, Stork O, Pape HC (2003). Amygdalar and hippocampal theta rhythm synchronization during fear memory retrieval. Science (New York, NY) 301: 846-850.

Shepard PD, Holcomb HH, Gold JM (2006). Schizophrenia in translation: the presence of absence: habenular regulation of dopamine neurons and the encoding of negative outcomes. Schizophr Bull 32: 417-421.

Shumake J, Ilango A, Scheich H, Wetzel W, Ohl FW (2010). Differential neuromodulation of acquisition and retrieval of avoidance learning by the lateral habenula and ventral tegmental area. J Neurosci 30: 5876-5883.
Tomimoto H, Kamo H, Kameyama M, McGeer PL, Kimura H (1987). Descending projections of the basal forebrain in the rat demonstrated by the anterograde neural tracer Phaseolus vulgaris leucoagglutinin (PHA-L). Brain Res 425: 248-255.

Ullsperger M, von Cramon DY (2003). Error monitoring using external feedback: specific roles of the habenular complex, the reward system, and the cingulate motor area revealed by functional magnetic resonance imaging. J Neurosci 23: 4308-4314.

Wang DG, Gong N, Luo B, Xu TL (2006). Absence of GABA type A signaling in adult medial habenular neurons. Neuroscience 141: 133-141.

Winson J (1978). Loss of hippocampal theta rhythm results in spatial memory deficit in the rat. Science (New York, NY) 201: 160-163.

Winter C, Vollmayr B, Djodari-Irani A, Klein J, Sartorius A (2011). Pharmacological inhibition of the lateral habenula improves depressive-like behavior in an animal model of treatment resistant depression. Behav Brain Res 216: 463-465.

Womelsdorf T, Schoffelen JM, Oostenveld R, Singer W, Desimone R, Engel AK et al (2007). Modulation of neuronal interactions through neuronal synchronization. Science (New York, NY) 316: 1609-1612.

Zagami MT, Ferraro G, Montalbano ME, Sardo P, La Grutta V (1995). Lateral habenula and hippocampal units: electrophysiological and iontophoretic study. Brain Res Bull 36: 539-543.

Supplementary Information accompanies the paper on the Neuropsychopharmacology website (http://www.nature.com/npp) 